\title{
Supporting Information \\ Role of Aspect Ratio in the Photoluminescence of Single CdSe/CdS Dot-in-Rods
}

Xiuqing Bai, ${ }^{\text {a,\# }}$ Huiyu Li, ${ }^{\text {b,\# }}$ Yonggang Peng, ${ }^{\text {c }}$ Guofeng Zhang, ${ }^{\text {a,* }}$ Changgang Yang, ${ }^{\text {a Wenli }}$

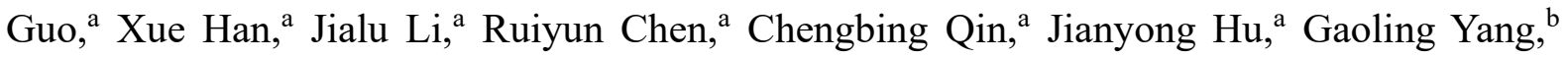
Haizheng Zhong, ${ }^{\mathrm{b}}$ Liantuan Xiao, ${ }^{\mathrm{a}, *}$ and Suotang $\mathrm{Jia}^{\mathrm{a}}$

atate Key Laboratory of Quantum Optics and Quantum Optics Devices, Institute of Laser Spectroscopy, Collaborative Innovation Center of Extreme Optics, Shanxi University, Taiyuan, 030006, China

${ }^{\mathrm{b}}$ Beijing Key Laboratory of Nanophotonics and Ultrafine Optoelectronic Systems, School of Materials Science and Engineering, Beijing Institute of Technology, Beijing, 100081, China ${ }^{c}$ School of Physics, Shandong University, Jinan 250100, China

${ }^{\#}$ Equal contribution

${ }^{*}$ Corresponding Authors.

E-Mail: guofeng.zhang@sxu.edu.cn (G. Z.)

xlt@sxu.edu.cn (L. X.) 


\section{S1. Estimation of the excitation condition $<N>$ value}

The excitation condition $\langle N\rangle$ value of single nanocrystal can be estimated by the absorption cross-section $(\sigma)$ of individual nanocrystal and the excitation photon flux $(j)$. The absorption cross-section $\sigma$ can generally be determined by fitting the PL saturation curve with the equation $y=a\left(1-e^{-\sigma j}\right),{ }^{1}$ as shown in Figure S1. The laser pump fluence $j=\frac{P}{F E}$, where $P$ is the laser power density, $F$ is the laser repetition rate, and $E$ is the laser photon energy. According to the values of $\sigma$ and $j$, the $\langle N\rangle$ value can be obtained by $\langle N\rangle=\sigma j$.

\section{S2. Discussion on the reduced PL lifetime}

According to the band-edge exciton fine structure in Figure 6d, the dark-bright exciton splitting of the three DR samples does not exceed $5 \mathrm{meV}$. The dark-bright exciton splitting is the energy difference between the lowest fine-structure level $\left(\left|0^{\mathrm{L}}\right\rangle\right.$ or $\left.| \pm 2\rangle\right)$ that is always optically inactive and the first optically active level $\left(\left| \pm 1^{\mathrm{L}}\right\rangle\right)$. At room temperature, the thermal energy $\left(k_{B} T\right)$ is $\sim 25 \mathrm{meV}^{2}$ The dark-bright exciton splitting of $5 \mathrm{meV}$ is much less than the thermal energy of $25 \mathrm{meV}$. In this case, the bright and dark exciton states are thermally mixed. The thermal mixing of bright and dark exciton states has little effect on the PL lifetime. ${ }^{3}$

For the three CdSe/CdS DR samples used in our work, the average widths of CdS rod are $4.2 \mathrm{~nm}, 4.2 \mathrm{~nm}$, and $4.4 \mathrm{~nm}$, respectively, and the average lengths of CdS rod are $30 \mathrm{~nm}, 60$ $\mathrm{nm}$, and $180 \mathrm{~nm}$, respectively. According to the shape anisotropy of the three DR samples, the dielectric effects of the three DR samples are theoretically calculated to be $2.7,3.1$, and 3.2 (see $\mathbf{S 4}$ for details). The slight increase in the dielectric effects indicates a slight increase in the dielectric screening effect of the three DR samples. According to recent reports, ${ }^{4,5}$ the enhancing dielectric screening effect can increase the PL lifetime of excitons. However, the averaged PL lifetimes (bright states) reduce from 32.7 to $10.1 \mathrm{~ns}$ with the increasing aspect ratios, as shown in Figure 2d. Therefore, the dielectric screening effect has a negligible influence on the PL lifetime.

For the single-dot measurements, the PLQY of bright state of PL intensity trajectory is generally considered to be close to unity. Therefore, exciton recombination in the bright state 
is just radiative recombination, and corresponding PL lifetime can be determined by radiative recombination rate $\left(\tau=\frac{1}{k_{r}}\right)$. When the PL lifetime decreases, the radiative recombination rate increases. The increased radiative rate may be due to an increase in the overlap of electronhole wave functions. We speculate that the change in the overlap of electron-hole wave functions may originate from the three $\mathrm{CdSe} / \mathrm{CdS} \mathrm{DR}$ samples with both type I and quasi-type II band alignments. ${ }^{6}$ The DR1 with a relatively small CdSe core may be the quasi-type II DRs, while both DR2 and DR3 with a relatively large core may be the type I DRs. Therefore, DR1 has the larger PL lifetime than that of DR2 and DR3. However, this is just a speculation. After all, the difference in their average core diameters is relatively small.

\section{S3. Blinking mechanism determined by radiative lifetime scaling.}

The radiative lifetime scaling for single DR1, DR2 and DR3 are calculated as follows. Three typical PL trajectories for a single DR1, a single DR2 and a single DR3 are presented in Figure S3a-c. Corresponding PL decay curves obtained from the PL regions marked in respective colors on PL intensity trajectories, as shown in Figure S3d-f. According to the PL intensity and lifetime values, the radiative lifetime scaling for single DR1, DR2 and DR3 can be calculated by the following equations.

$$
\begin{gathered}
\tau_{r 1}: \tau_{r 2}=\frac{\tau_{1}}{I_{1}}: \frac{\tau_{2}}{I_{2}}=\frac{31.4}{1165-10(\text { background })}: \frac{3.15}{128-10(\text { background })} \approx 1.02 \\
\tau_{r 1}: \tau_{r 2}=\frac{\tau_{1}}{I_{1}}: \frac{\tau_{2}}{I_{2}}=\frac{10.28}{619-10(\text { background })}: \frac{2.12}{133-10(\text { background })} \approx 0.98 \\
\tau_{r 1}: \tau_{r 2}=\frac{\tau_{1}}{I_{1}}: \frac{\tau_{2}}{I_{2}}=\frac{9.82}{352-10(\text { background })}: \frac{2.21}{88-10(\text { background })} \approx 1.01
\end{gathered}
$$

The radiative lifetime scaling is calculated to be $\sim 1.0$ for single DR1, DR2 and DR3, indicating the blinking mechanism of DR1, DR2 and DR3 to be the BC-blinking.

\section{S4. Calculation of the dielectric effect factor $R_{e}$.}

According to References ${ }^{2,7-9}$, the dielectric effect factor $R_{\mathrm{e}}$ of DR can be written: 


$$
R_{e}=\frac{f_{c}}{f_{a}}
$$

Where $f_{c}$ is the ratio of electric field amplitude inside the nanorod over the electric field amplitude outside nanorod along the nanorod,

$$
f_{c}=\frac{E_{c}(\text { in })}{E_{c}(\text { out })}=\frac{\varepsilon_{\text {medium }}}{\varepsilon_{\text {medium }}+\left(\varepsilon_{\text {rod }}-\varepsilon_{\text {medium }}\right) \alpha_{c}} .
$$

$f_{a}$ is the ratio of electric field amplitude inside the nanorod over the electric field amplitude outside nanorod perpendicularly to the nanorod,

$$
f_{a}=\frac{E_{a}(\text { in })}{E_{a}(\text { out })}=\frac{\varepsilon_{\text {medium }}}{\varepsilon_{\text {medium }}+\left(\varepsilon_{\text {rod }}-\varepsilon_{\text {medium }}\right) \alpha_{a}} .
$$

In equations (5) and (6), $\alpha_{c}$ and $\alpha_{a}$ are the depolarization factors along the nanorod and perpendicular to the nanorod, respectively.

$$
\begin{gathered}
\alpha_{c}=\frac{1-e^{2}}{2 e^{3}}\left(\ln \left(\frac{1+e}{1-e}\right)-2 e\right), \\
\alpha_{a}=\frac{1}{2}\left(1-\alpha_{c}\right),
\end{gathered}
$$

where $e$ represents the average aspect ratio (rod length/rod width) for DRs.

Finally,

$$
R_{e}=\frac{f_{c}}{f_{a}}=\frac{\varepsilon_{\text {medium }}+\left(\varepsilon_{\text {rod }}-\varepsilon_{\text {medium }}\right) \alpha_{a}}{\varepsilon_{\text {medium }}+\left(\varepsilon_{\text {rod }}-\varepsilon_{\text {medium }}\right) \alpha_{c}} .
$$

In our case, $\varepsilon_{\text {rod }}=\varepsilon_{C d S}=5.5$, and $\varepsilon_{\text {medium }}=\varepsilon_{\text {air }}=1$. The dielectric effect factor $R_{\mathrm{e}}$ as a function of aspect ratio is shown in Figure S5. It is found that as the aspect ratio increases, the dielectric effect factor $R_{\mathrm{e}}$ tends to be constant. Therefore, the contribution of the dielectric effect to the degree of emission polarization will tend to be constant. 


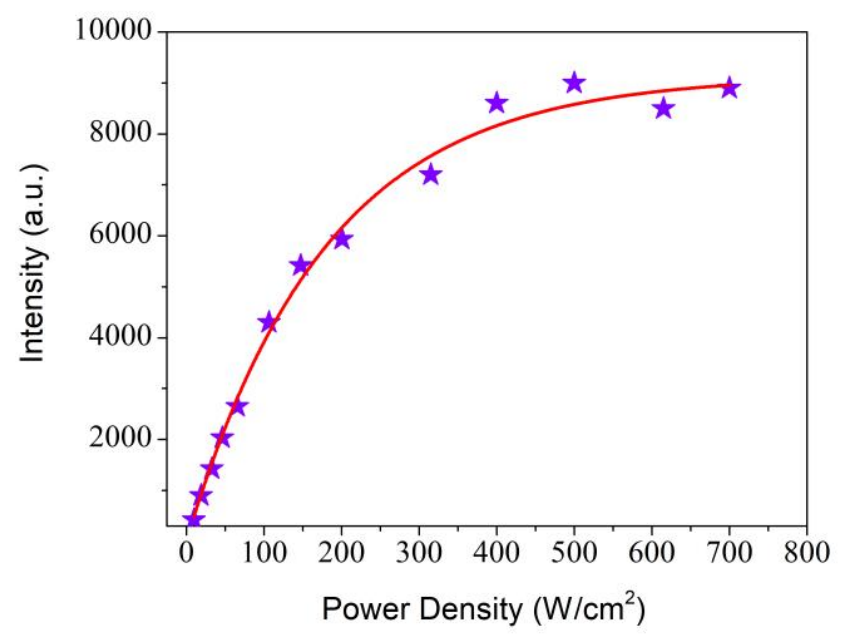

Figure S1. Typical PL saturation curve for single DR. The excitation condition $\langle N\rangle$ can be determined by fitting the PL saturation curve with the equation $y=a\left(1-e^{-\sigma j}\right)$.
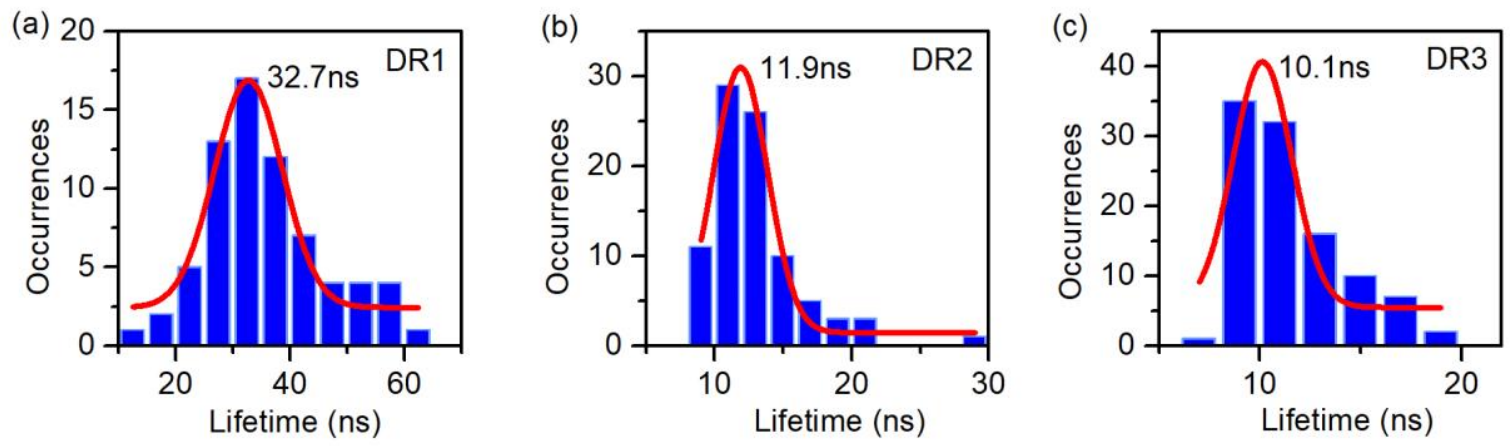

Figure S2. Histograms of PL lifetimes of bright-states for single DR1s (a), DR2s (b) and DR3s (c), respectively. Red curves are the corresponding Gaussian fits. 

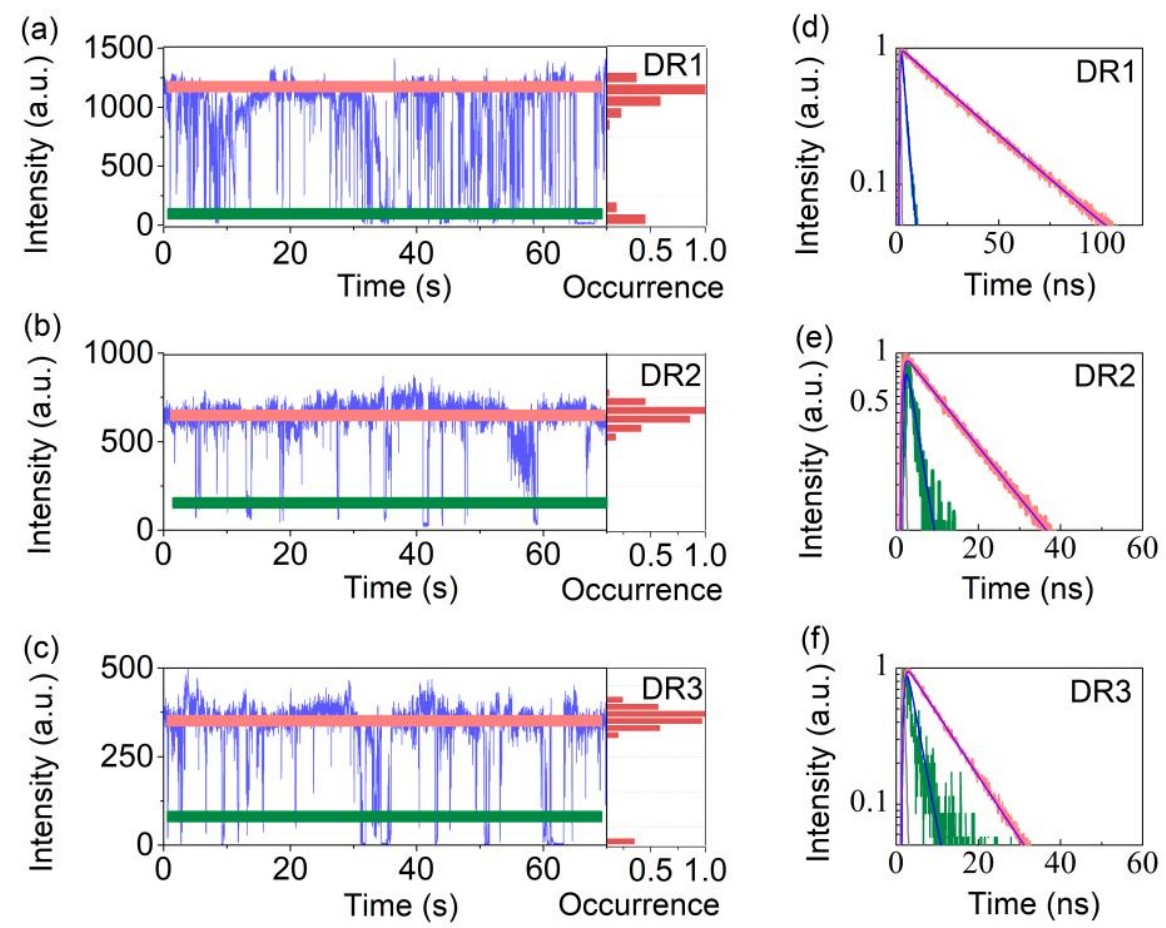

Figure S3. (a, b, c) Typical PL intensity trajectories for a single DR1, a single DR2 and a single DR3. (d, e, f) Corresponding PL decay curves obtained from the PL regions marked in respective colors on PL intensity trajectories of (a, b, c), respectively. 

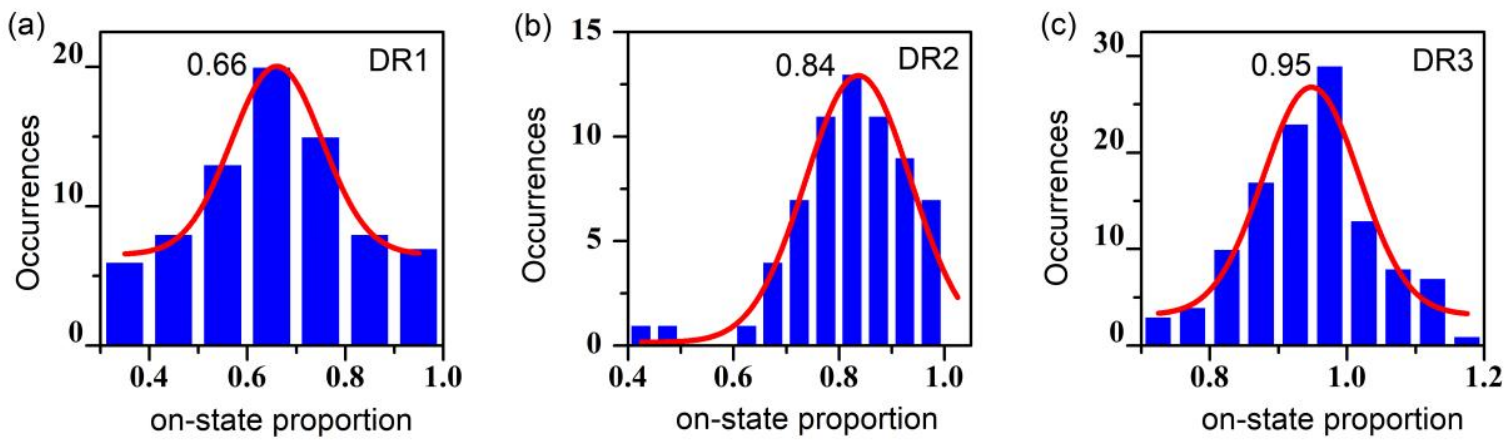

Figure S4. Histograms of the on-state proportions for single DR1s (a), DR2s (b) and DR3s (c), respectively. Red curves are the corresponding Gaussian fits.

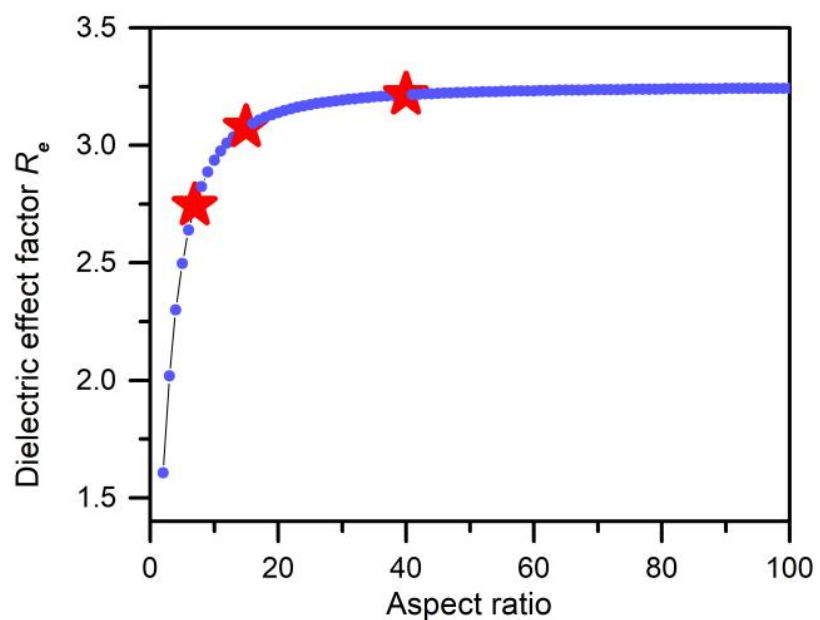

Figure S5. Dielectric effect factor $R_{\mathrm{e}}$ as a function of aspect ratio. Single DRs with the aspect ratios of 7.1, 14.3 and 40.9 correspond to DR1, DR2 and DR3, respectively, as indicated by the red stars. 


\section{References:}

(1) Hu, F. R.; Zhang, H. C.; Sun, C.; Yin, C. Y.; Lv, B. H.; Zhang, C. F.; Yu, W. W.; Wang, X. Y.; Zhang, Y.; Xiao, M. Superior Optical Properties of Perovskite Nanocrystals as Single Photon Emitters. ACS Nano 2015, 9, 12410-12416.

(2) Vezzoli, S.; Monceau, M.; Lemenager, G.; Glorieux, Q.; Giacobino, E.; Carbone, L.; De Vittorio, M.; Bramati, A. Exciton Fine Structure of CdSe/CdS Nanocrystals Determined by Polarization Microscopy at Room Temperature. ACS Nano 2015, 9, 7992-8003.

(3) Crooker, S. A.; Barrick, T.; Hollingsworth, J. A.; Klimov, V. I. Multiple Temperature Regimes of Radiative Decay in CdSe Nanocrystal Quantum Dots: Intrinsic Limits to the DarkExciton Lifetime. Appl. Phys. Lett. 2003, 82, 2793-2795.

(4) Hou, X. Q. Q., H. Y.; Peng, X. G. Enhancing Dielectric Screening for Auger Suppression in CdSe/CdS Quantum Dots by Epitaxial Growth of ZnS Shell. Nano Lett. 2021, 21, 38713878.

(5) Hou, X. Q.; Kang, J.; Qin, H. Y.; Chen, X. W.; Ma, J. L.; Zhou, J. H.; Chen, L. P.; Wang, L. J.; Wang, L. W.; Peng, X. G. Engineering Auger Recombination in Colloidal Quantum Dots Via Dielectric Screening. Nat. Commun. 2019, 10, 1750.

(6) Wu, K.; Hill, L. J.; Chen, J.; McBride, J. R.; Pavlopolous, N. G.; Richey, N. E.; Pyun, J.; Lian, T. Universal Length Dependence of Rod-to-Seed Exciton Localization Efficiency in Type I and Quasi-Type II CdSe@CdS Nanorods. ACS Nano 2015, 9, 4591-4599.

(7) Sihvola, A. Dielectric Polarization and Particle Shape Effects. J. Nanomater. 2007, 2007, 
45090.

(8) Cassette, E.; Mahler, B.; Guigner, J. M.; Patriarche, G.; Dubertret, B.; Pons, T. Colloidal CdSe/CdS Dot-in-Plate Nanocrystals with 2D-Polarized Emission. ACS Nano 2012, 6, 67416750.

(9) Kamal, J. S.; Gomes, R.; Hens, Z.; Karvar, M.; Neyts, K.; Compernolle, S.; Vanhaecke, F. Direct Determination of Absorption Anisotropy in Colloidal Quantum Rods. Phys. Rev. B 2012, $85,035126$. 